\title{
Research on the Key Technique of Wireless Network Systems Based on ZigBee and Related Techniques
}

\author{
LIN $\operatorname{Nan}^{1, a}$ \\ ${ }^{1}$ College of Software Technology, Zheng Zhou University, Henan Zhengzhou, 450002, China \\ a linnan@zzu.edu.cn
}

Keywords: Wireless Network Systems; ZigBee; Key Technique; Hardware Design and Verification.

\begin{abstract}
In this paper, we did research on the key technique of wireless network systems based on ZigBee and related techniques. ZigBee wireless sensor network is composed of multiple interconnected ZigBee nodes through wireless channel which supports three types of network topology star, tree, and mesh. Although figure of the wireless link with that line but the truth is nearby nodes are under its radio coverage which can form depends on the network routing algorithm used in the wireless link. Our proposed system solve the corresponding issues successfully.
\end{abstract}

\section{Introduction}

ZigBee is an emerging short-range, low-rate wireless network technology, wireless sensor networks, and other occasions, a wide range of applications. ZigBee device power consumption and costs are low, so you can ensure that the ZigBee-based positioning system can achieve long accurate positioning at a lower price. Cognitive radio technology has become a new train of thought on the development of wireless communication and research hot spot. The core idea of cognitive radio technology is which has the function of cognitive radio communication equipment through wireless environment perception authorization frequency spectrum hole and the current study reasoning update existing knowledge base. Thus make intelligent behavior prediction and adaptively adjust the optimal transmission strategy and the implementation will include sharing has authorized spectrum hole, thus effectively improve spectrum efficiency and alleviate the status of the current spectrum resource scarcity. Sensor nodes are the basic building blocks of wireless sensor network unit which is mainly responsible for the surrounding information acquisition and processing, and send the data to adjacent nodes or adjacent nodes forward sending data to coordinate nodes or nodes closer to coordinate. The nodes of wireless sensor network usually consist of sensor module, processor module, wireless communication module and power module. In network driven approach, the network through the centralized management, can the network resource allocation to a certain area or specified user switching to a specific network. This kind of method of defect is in the system between various wireless accessing networks must tightly integrated together and a large number of additional signaling overhead may be produced. Another user driven approach, through mobile user could active network selection decisions or vertical switching implementation. Based on the user driven way due to the low implementation complexity and less overhead are relatively easy to achieve but the user must carry on the decision-making must first obtain a certain amount of information. If the user access to all the current state of the network information, we can choose the optimal network or switching decisions but often it is hard to get the network state information. ZigBee technology is a short-range, low power, low data rate; low-cost two-way wireless communication technology can be embedded into various devices, and support geo-targeting capabilities. ZigBee technology used in wireless sensor network is a focus of the present study and targeted research and application of technology has also been widespread concerned [1-4].

Game theory refers to the study of multiple between individuals or groups, in particular condition under the restriction of the use of related party game strategy, implement the corresponding strategy of discipline is the study is in the nature of struggle or competition phenomenon. The theory and methods of game theory in many fields can be as important theoretical tool to solve the problem of the 
conflict and cooperation such as biology, economics, international relations, computer science, politics, military strategy, etc. In recent years, the game theory is also used in the design and analysis of wireless communication network system including the power control, resource allocation, routing and load balancing, flow control, admission control and ask. Therefore, in this paper, we undertake theoretical analysis on the key technique of wireless network systems based on ZigBee and related techniques which will be discussed in detail in the following sections.

\section{The Principle Techniques}

The Topology of the ZigBee Structure. It could not directly observe the structure of the ZigBee Wireless Sensor Network as the wired networks. This problem makes it difficult to manage and control the ZigBee WSN. ZigBee wireless sensor network is composed of multiple interconnected ZigBee nodes through wireless channel which supports three types of network topology star, tree, and mesh. Although figure of the wireless link with that line but the truth is nearby nodes are under its radio coverage which can form depends on the network routing algorithm used in the wireless link. In a star network structure, ZigBee coordinator is the center of the whole network and it is responsible for establishing, managing and maintaining the whole network. At the same time, all the other nodes only through the coordinator to join the network and the network node can only communicate and coordinator. Mesh network structure is the most general structure and its routing can automatically set up and maintenance. Nodes in a network of equal status can communicate with all nodes within the scope of direct communication. But as a result of a node in a certain moment can only choose a link communication, won't form a routing loop, so we can think it is still a tree structure network. In the figure 1, we show the mentioned topology of the ZigBee structure.

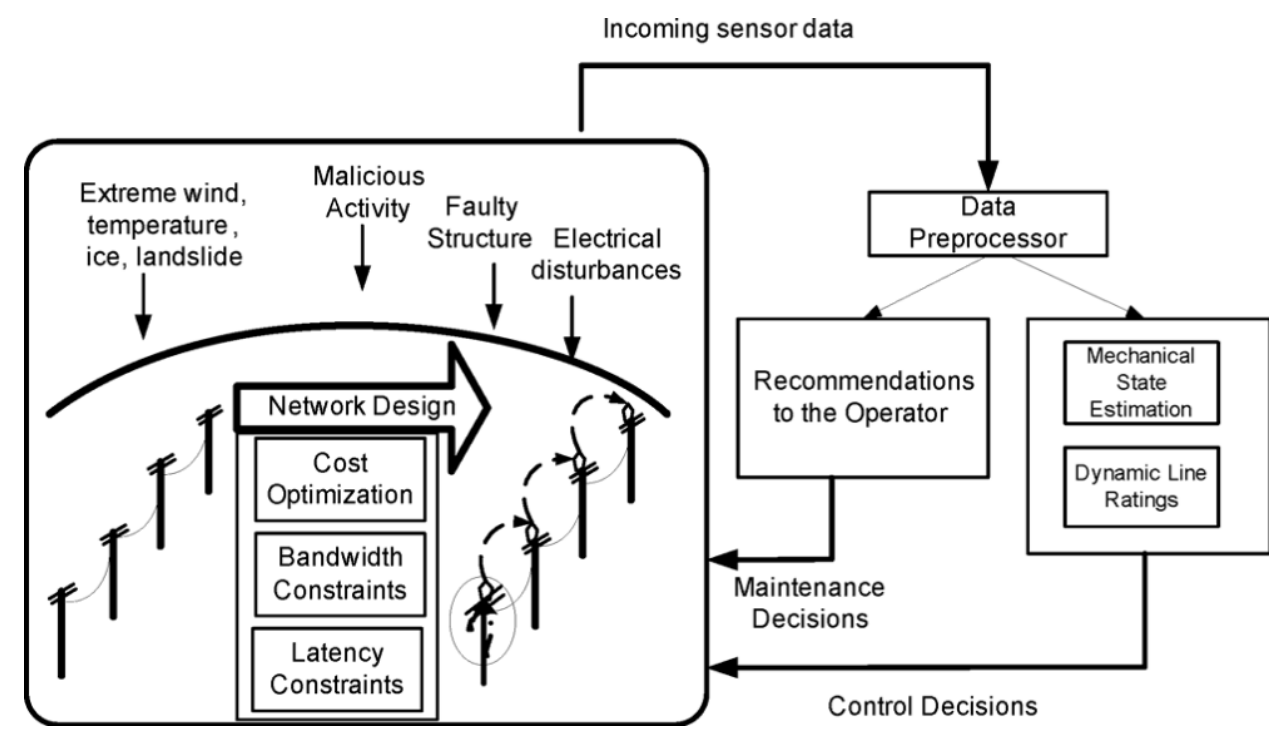

Fig. 1The Topology of the Mentioned ZigBee Structure

ZigBee network differs from that of wired network which cannot directly observe the whole network of the network and the formation of the network topology. To easily manage and control the ZigBee network, it must be based on a certain topology discovery algorithm using the topology of the networking measurement technology for ZigBee network. Through the ZigBee network networking process and principle of network, whether it be a star, tree, or a mesh network and all kinds of ZigBee network is initialized by the coordinator and establish the ZigBee router or terminal as well as by the coordinator to join the network but can be by ZigBee router to join the network. Star network and tree network topology structure, its spanning tree topology and mesh network spanning tree topology reflects the father-son relationship structure between the network nodes. Though it is not a complete network topology but reflects the core of the network structure. Therefore, by monitoring the topology spanning tree, we can observe state of each node in the network so that they can easily manage and control the entire network. In the next sections we will discuss in detail. 
The Wireless Network System Implementation. Composed of wireless sensor networks deployed near the observation environment of a large number of micro-cheap low-power sensor nodes, sensing and data processing and wireless communication capabilities through the formation of multi-hop self-organizing wireless network systems we could implement the corresponding system. It is independent of the base station to mobile routers and other communications infrastructure, since a network of distributed protocols. For ZigBee network topology, we first need to know the information in the network node, i.e. what are the active nodes in the network and its basic information and then need to know the relationship between the nodes in a network. Therefore, this article design ZigBee network found topology algorithm basic idea is to the ZigBee coordinator for all the basic information of the active node in the network and put it in to a group called Nodes in the array, then traverse the Nodes each element of the array and send commands to the ZigBee network asked each storage node's parent address after get the parent address, the array to find the node address is the same as the address of the parent element. Structural health monitoring is divided into the overall monitoring and local monitoring. Regardless of partial or whole monitoring, accurate data collection and transmission in sensor for the premise and foundation and all kinds of structural health monitoring data acquisition mainly adopts the traditional sensor to achieve wired shown in the following figure.

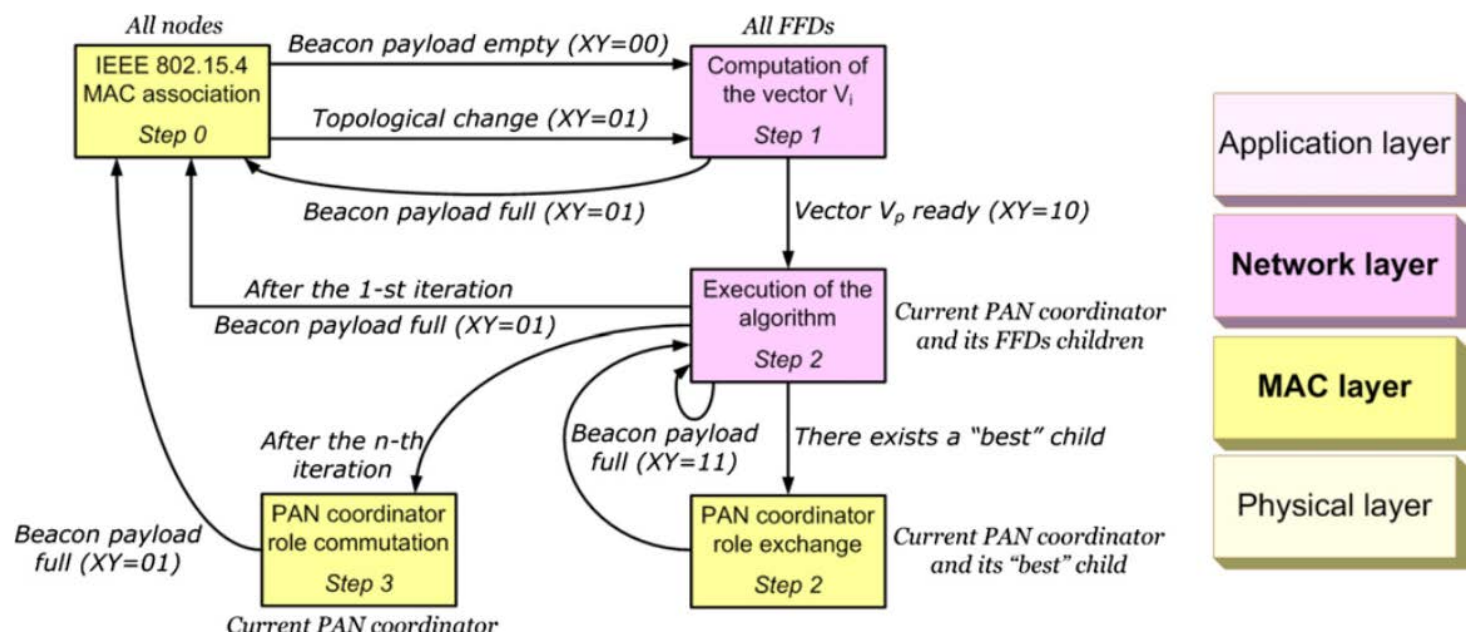

Fig. 2The Logistics and Implementation of Wireless Network System

Network technology such as wireless local area network (LAN) barter information collected and transmitted to the intelligence gathering gateway through intelligence gathering gateway access to the network integration system and finally uses including radio and television networks. A huge number of sensor nodes deployed in the form of random spread or artificial placed in monitoring area, build the network by way of self-organization. By sensor nodes through the network monitoring to the area of data in multiple hops routing nodes in the transmission to gathering node, the data could be in transit for multiple nodes perform fusion and compression, finally, Internet or wireless access the server via satellite terminal management node. At the same time, the end user can through the subjective behavior affect the underlying network geared to the needs of different applications so as to realize united information interaction between people and things.

The Related Standard and Agreement. Adopting a cross-layer approach, the proposed algorithm operates at the network layer of a WSN that relies on the IEEE 802.15.4 MAC layer for the network formation, as represented in Fig. 3. The topology of a WSN formed according to the IEEE 802.15.4 MAC layer is a cluster-tree where the PAN coordinator is at the root of this tree. 


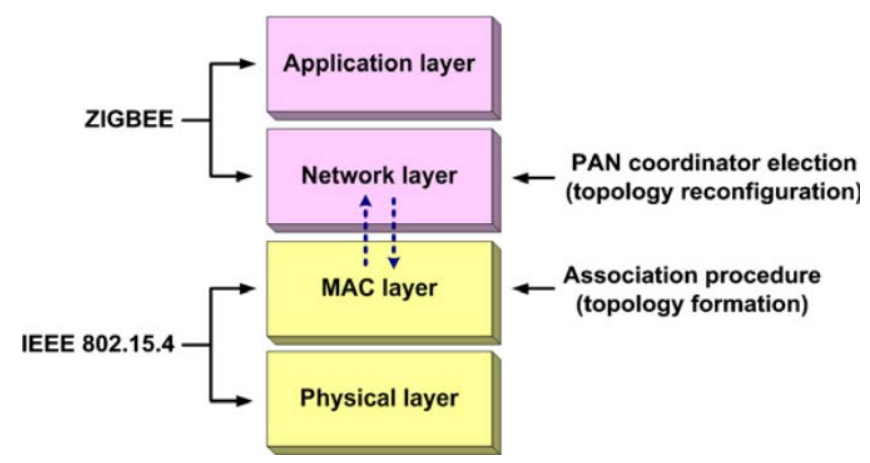

Fig. 3The Related Standard and Agreement for the System

Based on the structure of the cluster tree network modeling while provides an overall and flexible framework but the node function of the model is stratified. Cluster head nodes usually transport a large number of data packets and energy consumption significantly. In such cases, the network node of inequality will reduce the life cycle of the network. The standard defines the steps taken by p and the other nodes to initialize and form the network, respectively. Node p starts by selecting a suitable communication channel. This selection is performed by the energy detection scan which assesses the level of interference on each channel by measuring the peak energy on each available channel. In the next section we will show the verification result for the proposed system.

\section{Experiment and Verification}

In the paper, QT4.6.0 is used for developing monitoring center software system. QT belongs to a cross-platform $\mathrm{C}++$ graphical user interface library. It has good cross-platform performance, which can be operated on embedded platform. In the following figure 4 we show the result.

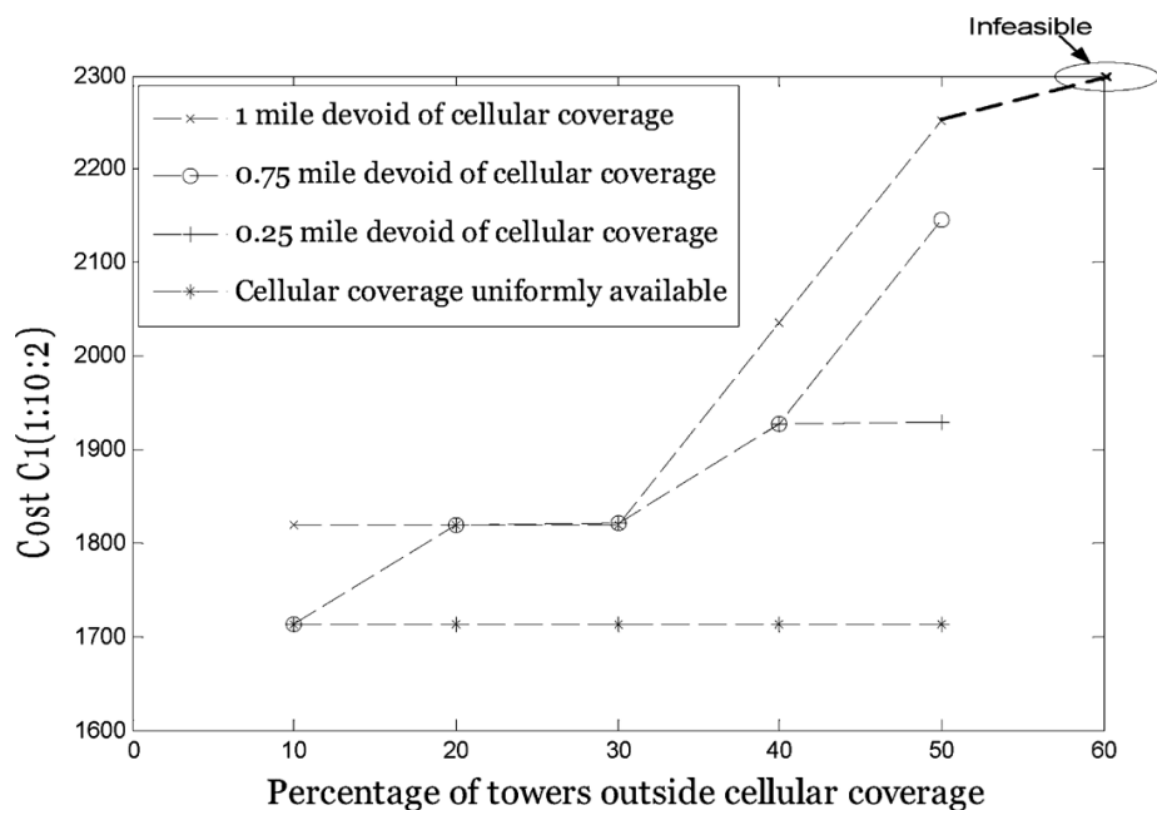

Fig. 4The Verification Result for the System

\section{Conclusion and Summary}

Sensor nodes are the basic building blocks of wireless sensor network unit which is mainly responsible for the surrounding information acquisition and processing and send the data to adjacent nodes or adjacent nodes forward sending data to coordinate nodes or nodes closer to coordinate. In this paper, we did research on the key technique of wireless network systems based on ZigBee and related techniques. The result proves the feasibility of our method. We plan to modify the current communication standard among ZigBee devices in the near future. 


\section{References}

[1] Jiayang L, Wenxiang Z. Design of and Research on Wireless Network's Universal Data Transmission System Based on ZigBee[J]. Sci Tech Information Development \& Economy, 2014.

[2] Huang Y M, Hsieh M Y, Chao H C, et al. Pervasive, secure access to a hierarchical sensor-based healthcare monitoring architecture in wireless heterogeneous networks[J]. Selected Areas in Communications, IEEE Journal on, 2009, 27(4): 400-411.

[3] Jun Z, Jun-zi H, Xiao-yan L, et al. Design of Emergent Equipment System Based on ZigBee Wireless Network in the Hospital[J]. China Medical Devices, 2014.

[4] [3]Lu Z, Li Y, Fu T. Intelligent Wind-speed Monitoring System Based on the Zigbee Wireless Network[J]. International Journal of Online Engineering, 2015, 11.

[5] Peng-hao X, Yu-guang F, Wen-jun X. Study on controllable network building based on ZigBee wireless network[J]. Electronic Design Engineering, 2014.

[6] ZHANG Z, XU X, YAN L. Underground localization algorithm of wireless sensor network based on Zigbee [J]. Journal of China Coal Society, 2009, 1: 028.

[7] Gungor V C, Hancke G P. Industrial wireless sensor networks: Challenges, design principles, and technical approaches[J]. Industrial Electronics, IEEE Transactions on, 2009, 56(10): 4258-4265. 